\title{
EXPERIMENTAL STUDIES OF SPRAY DEPOSITION ON A FLAT SURFACE IN A VACUUM ENVIRONMENT
}

\author{
Eric L. Golliher ${ }^{1 *}$, S. C. Yao ${ }^{2}$ \\ ${ }^{1}$ NASA Glenn Research Center, Cleveland, Ohio, 44135, USA \\ ${ }^{2}$ Carnegie Mellon University, Pittsburgh, PA 15213, USA
}

\begin{abstract}
Cooling of spacecraft components in the space environment is an on-going research effort. The electronics used in modern spacecraft are always changing and the heat flux is increasing. New, one-of-a-kind missions require new approaches to thermal control. In this research, under vacuum conditions, a pulsed water spray impinged on a small disc, while a high speed data acquisition system recorded the temperature histories of this copper disc. The water droplets froze quickly and accumulated on the disc as the spray continued. After the spray stopped, the frozen water that remained on the disc then sublimated into the vacuum environment and cooled the disc. This paper examines two important aspects of this process: 1) the difference in spray start up and shutdown in a vacuum environment versus in a standard atmospheric pressure environment, and 2) the water utilization efficiency in a vacuum environment due to the effects of drop trajectories and drop bouncing on the surface. Both phenomena play a role during spray cooling in a vacuum. This knowledge should help spacecraft designers plan for spray cooling as an option to cool spacecraft electronics, human metabolic generated heat, and heat from other sources.
\end{abstract}

KEY WORDS: Spray Cooling, Phase Change, Vacuum

\section{INTRODUCTION}

This research is related to the investigation of heat transfer from a flat plate using water spray cooling at system pressures below standard atmospheric pressure. The focus is on those system pressures below the triple point of water. There has been limited research in this area. At the triple point of water, the three phases are in equilibrium: liquid, vapor and solid, at $0.01^{\circ} \mathrm{C}$ and $612 \mathrm{~Pa}$ (4.6 Torr). An understanding of the physical processes of operating below the triple point is needed for thermal control systems of future space missions. There are also many possible applications of this research on earth.

The original interest in this research comes from a piece of Shuttle hardware called the Flash Evaporator System (FES). This hardware cools the Orbiter main coolant loop to $4{ }^{\circ} \mathrm{C}$ during certain mission phases. Although the FES has been mostly successful, some past shuttle missions have had failures of this hardware[1]. It is thought that unexpected icing caused the failure. These failures have resulted in mission delays and undesirable warm crew cabin temperatures[2]. As the ice sublimated slowly, some mission delays were on the order of one hour, which impacted the re-entry. Little understanding exists for the mechanism of failure or possible mitigation. 


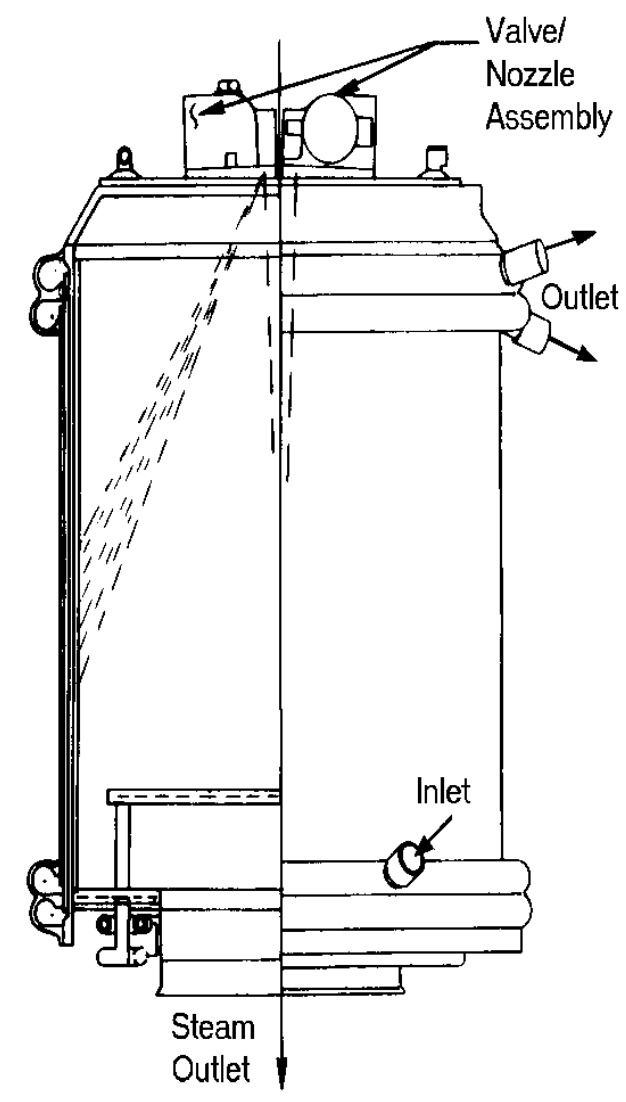

Figure 1 Shuttle Orbiter Flash Evaporator System -FES (O’Conner 2004)

The FES sprayed water on the inside of cylindrical compact heat exchanger which carried single phase coolant to other parts of the Orbiter, where waste heat was acquired from electronics, the cabin air, and other waste heat sources. A steam outlet exhausted to the vacuum of space. Figure 1 shows a cut-away drawing of the FES with the Inlet and Outlet coolant loop interfaces and a depiction of the spray impinging on the wall[1]. Figure 2 shows the location of the FES relative to the other thermal control hardware. The FES used a restriction in the downstream steam outlet to create "backpressure" that would cause the system pressure within the FES to nominally rise above the triple point and avoid freezing. This passive device relied upon a minimum vapor mass flowrate to guarantee choking at the restriction. It is thought that the failures of the FES were caused by some blockage or other inability of the passive device to create the proper system pressure within the FES. The occasional and unexplainable failure of the FES has had negative consequences for the future replacement of the Shuttle: a less efficient type of evaporative cooler for the follow-on human spaceflight capsule has been selected. This device is called a sublimator, and is relatively inefficient on a mass basis in its use of water, for operational conditions of rapid on/off or short duration cooling mission phase. If a good understanding of the icing mechanism for a spray evaporative cooling system can be achieved, it is possible that next generation human-rated space vehicles may indeed have the option of using a more efficient spray evaporative cooling system, thereby saving vehicle weight for more payload items. This research may provide the ability to design a better Flash Evaporator System as an option for NASA's next human space transportation system.

The Shuttle spray evaporative system, known as the Flash Evaporator System (FES) was developed in the late 1970's by Hamilton Standard of Windsor Locks, Connecticut. Since very little was known about spray cooling at that time, the design process consisted of much testing and re-testing, but seems to have involved little fundamental understanding of the processes involved. Since that era, research results published in recent journals may help better understand low system pressure spray cooling process, and the associated failure modes of the shuttle FES. The present study is focused on the spray dynamics in the vacuum, which is vastly different than the conventional situations. 


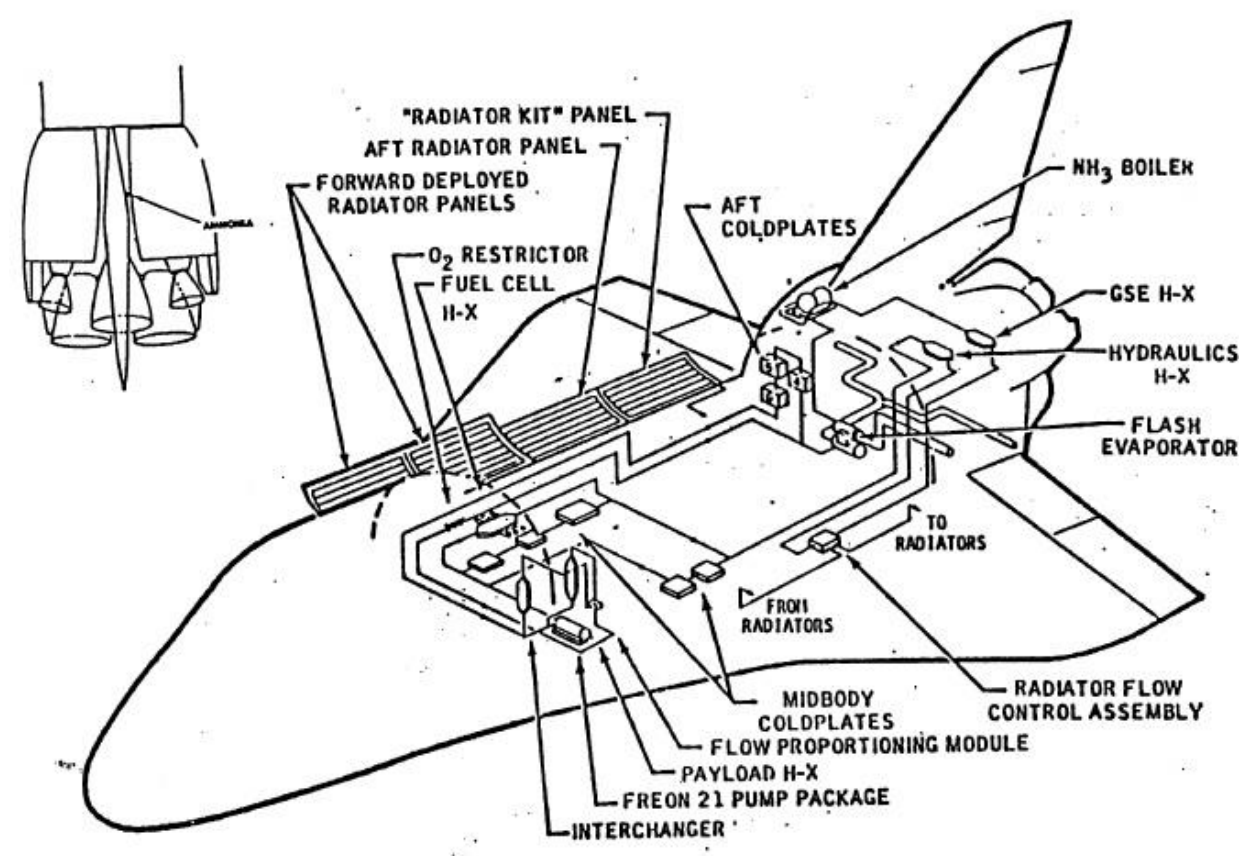

Figure 2 Shuttle Orbiter Thermal Control System

\section{EXPERIMENTAL SETUP}

A small chamber suitable for vacuum levels below the triple point was designed and built specifically for this research. Inside the chamber is a spray nozzle that impinges water onto the small copper disc. The chamber was designed to have both power and data feedthroughs, visible window ports, and a water line feed-through. This nozzle is composed of an automotive fuel injector, whereby the original atomizer is replaced with a very low flowrate Parker swirl nozzle. The spray pulse duration is controlled by a programmable digital controller that can switch the injector solenoid on and off with a pulse duration of as short as 8 milliseconds (ms). A laboratory vacuum pump is used to achieve system pressures as low as 0.04 Torr. The equilibrium ice temperature corresponding to this system pressure is about $-49 \mathrm{C}$.

The diagram in Figure 3 shows the overall setup and components. Two types of data are acquired: high speed video data and temperature history data. The pressure is reduced in the vacuum chamber by a small lab vacuum pump. There are two different references to "pressure": 1) the "nozzle pressure", which is the differential pressure across the nozzle that forces the water through the nozzle and 2) the "system pressure", which is the measure of vacuum pressure in the vacuum tank. The water is supplied to the nozzle by a plunger type system whereby air is pressurized on one side of the plunger and monitored with a pressure gauge. Water is filled on the other side of the plunger and thus, separate from the pressurized air. The spray nozzle solenoid controller is programmable to provide the length of pulse duration and start time. The digital data acquisition system is a high speed system capable of 6400 measurements per second of 5 channels. Figure 3 shows some additional details of the setup when working in the vacuum chamber. 


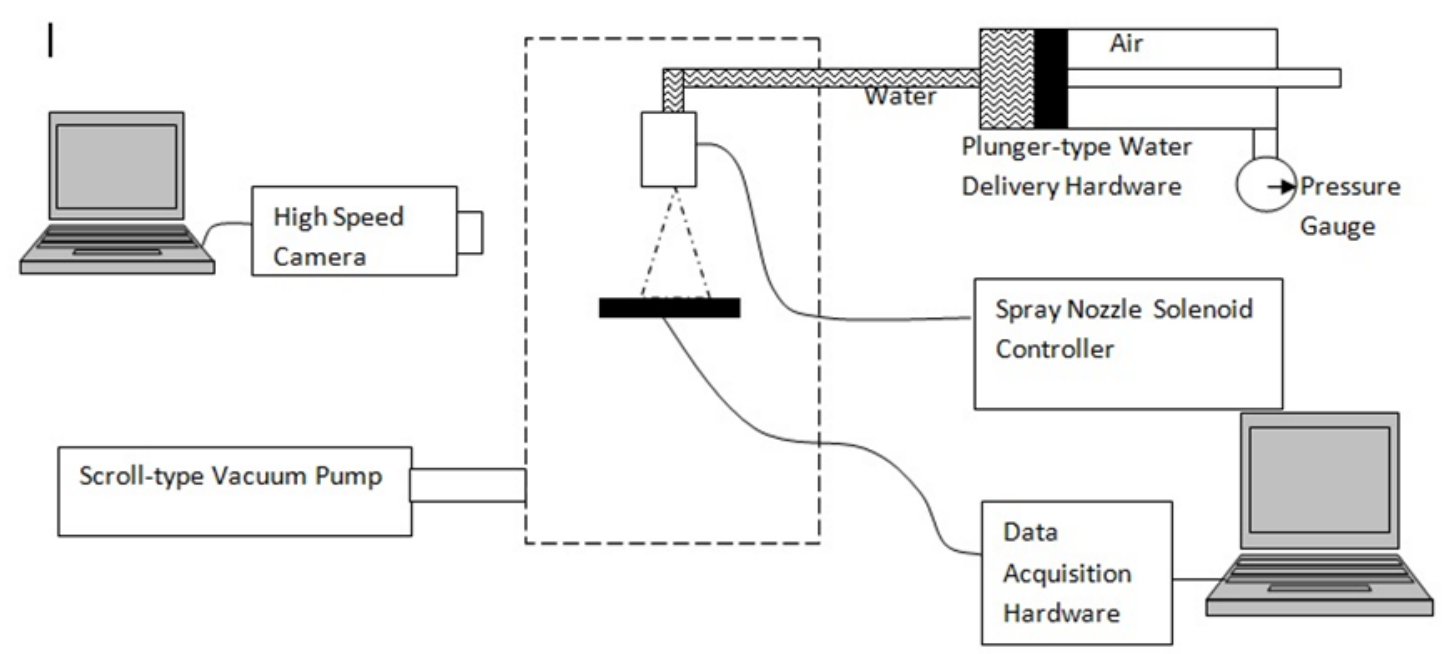

Figure 3 Experimental Setup

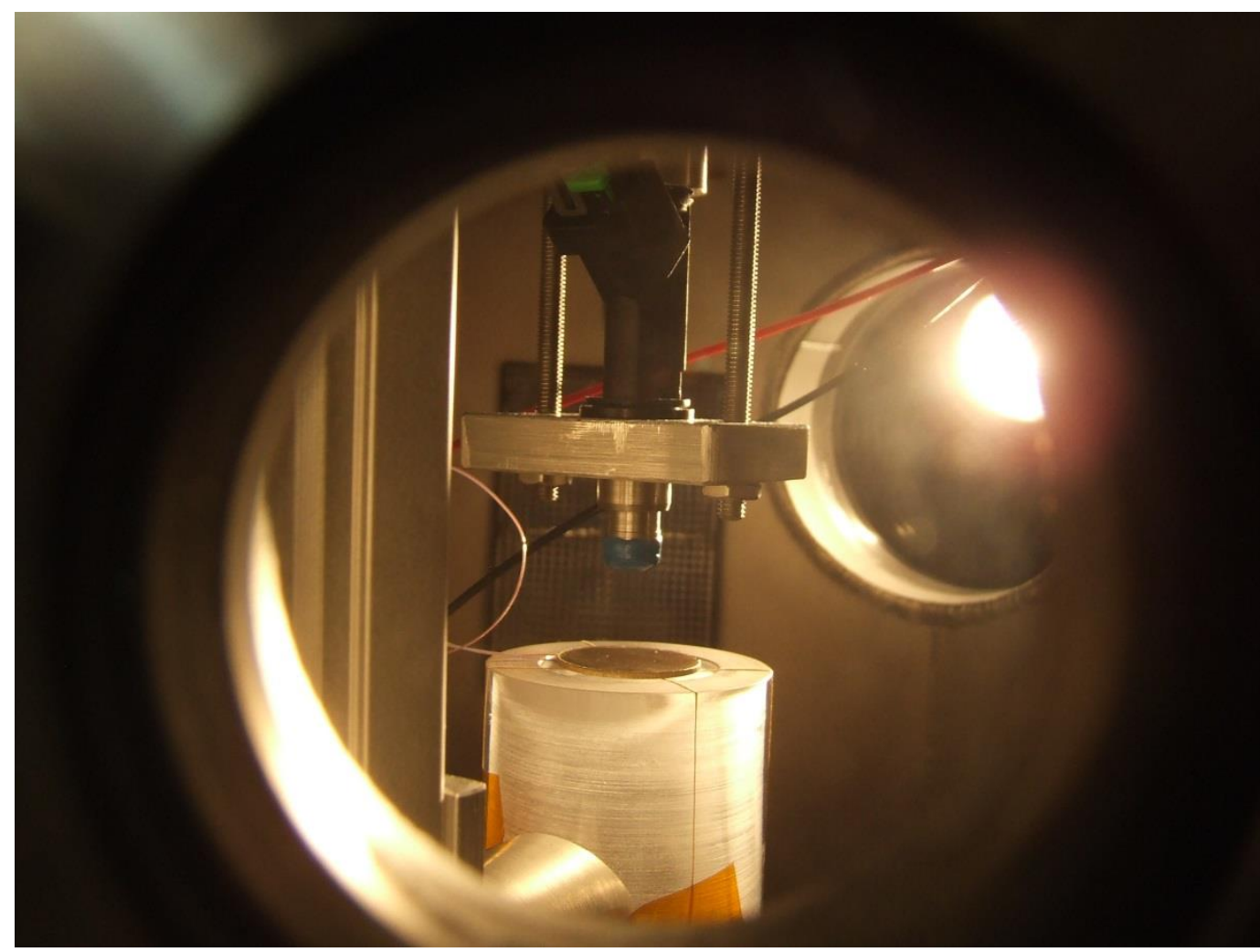

Figure 4 Vacuum Chamber View Showing Disc and Spray Nozzle

A Halogen lamp located outside of the chamber illuminated the disc as shown in Figure 4. The system pressure sensor is an MKS 629B1TQCJ2B capacitance manometer calibrated from 0 to 100 Torr over the output voltage range of $0-10 \mathrm{~V}$. The system pressure transducer voltage was converted to pressure [Torr] with an MKS PR4000 electronics unit. Also, the system pressure transducer raw voltage was displayed by a Keithly 2001 71/2 digit Multimeter. A capacitance manometer was chosen over other options, because it is 
ideal for this pressure range of near 0.01 Torr, and offers the highest degree of accuracy for this pressure range[3].

\section{EXPERIMENTAL RESULTS}

3.1 Nozzle Spray Startup in Vacuum. Due to the short duration of the pulsed spray, the startup feature of the nozzle in vacuum is important. Generally, the high speed video shows time of flight for the droplets to travel from the nozzle to the disc is between 1 and $2 \mathrm{~ms}$. For spray startup in air, there is some residual water held up in the nozzle that takes time to accelerate against the air resistance for good atomization. This is pronounced for all types of spray nozzles operating at nozzle pressures that are below their optimum design point, such as in this research, 25 psid. This effect is not important for most sprays because most sprays are steady sprays. Panao[4] is one of the few researchers that have recently studied the "leading front" and "tail" of a spray by using an advanced Phase Doppler Particle Analyzer (PDPA). He was able to quantify this effect and show its importance for the emerging field of high frequency pulsed spray cooling.

Figure 5 shows the time lapse process of spray start in air, taken with the high speed camera at 1000 frames per second (fps) and exposure of $996 \mathrm{~ns}$. The liquid that was never atomized is subsequently pushed out of the nozzle. A typical bell shape begins to form in Figure 6 at the nozzle tip, showing the beginning of successful atomization. Figure 10 is taken with an ordinary $35 \mathrm{~mm}$ camera and shows the fully developed spray in air for comparison.

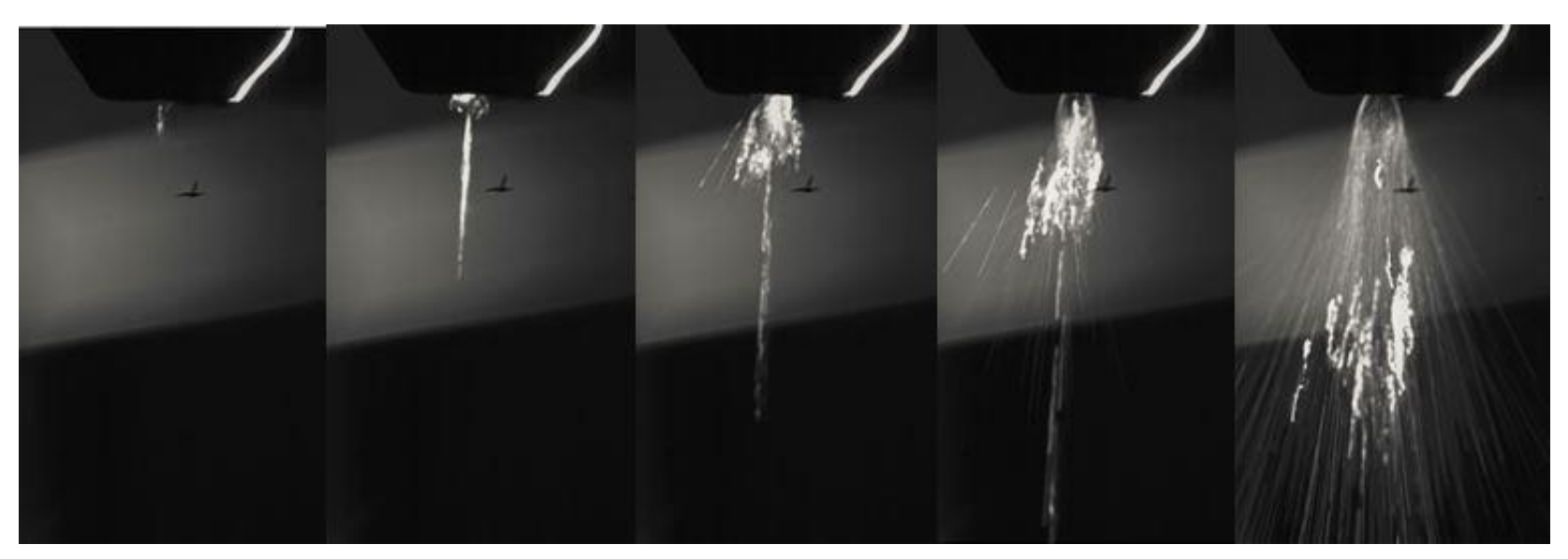

Figure 5 Spray Start Showing Increase Atomization and Unatomized Liquid time $=0$ to $5 \mathrm{~ms}$

In contrast to startup in air, startup in vacuum shows no signs of any unatomized liquid as shown in Figure 6. The spray appears to start immediately and atomization begins in less than $1 \mathrm{~ms}$, the frame rate of the camera. Although not shown, the end of the spray is exactly the same, with an apparent sharp cut-off. 


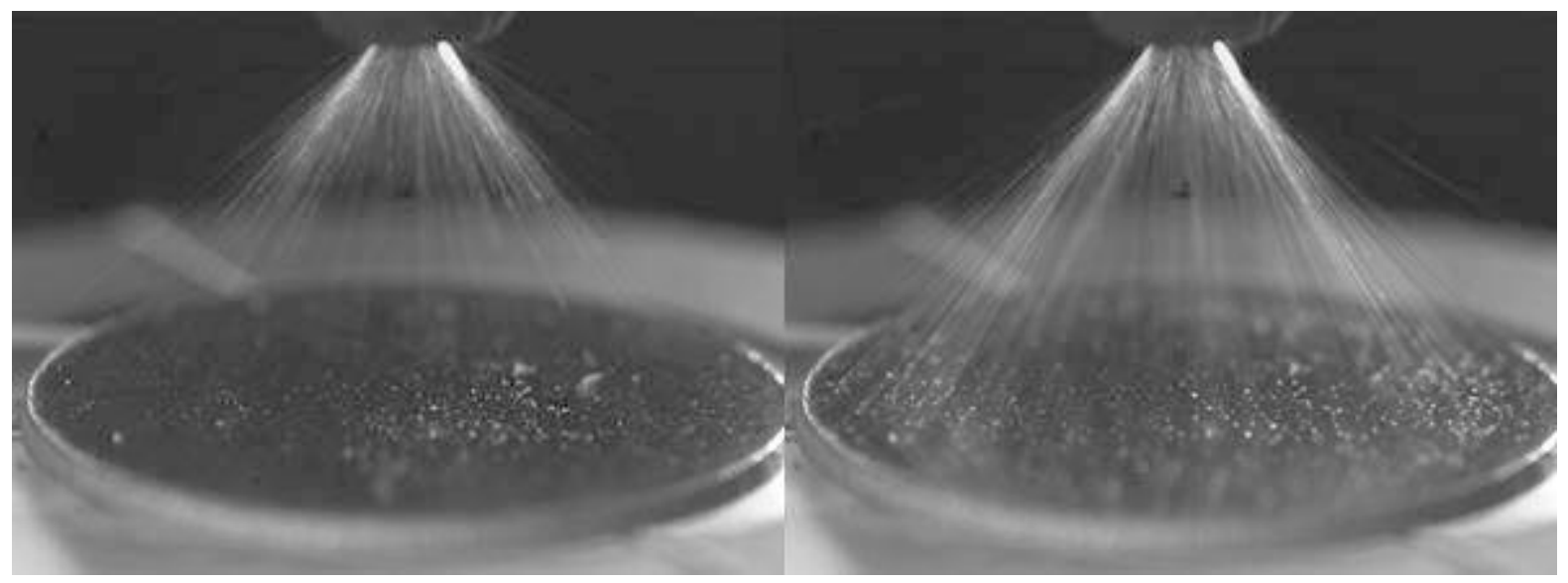

Figure 6 Spray Start in Vacuum $\mathrm{t}=0$ to $1 \mathrm{~ms}$

A possible explanation is that, without air in the nozzle chamber, the liquid entering the nozzle chamber from the four tangential ports can reach the working speed very fast. In addition, since there is no air in the vacuum chamber, there is no resistance and shear from the air to provide a retarding startup resistance.

3.2 Liquid Deposition Distribution. The process of atomization in air and in vacuum is quite different. In vacuum, the only atomization force is the turbulence in the nozzle. In air, the additional air shear force induces better atomization. In air, the spray pattern is a solid cone, which is also the specification provided by the vender of the nozzle, but in vacuum the spray pattern becomes a hollow cone as can be seen in Figure 7. The deposition of sprayed droplets on the disc exhibits a ring pattern owing to the hollow cone-type spray.
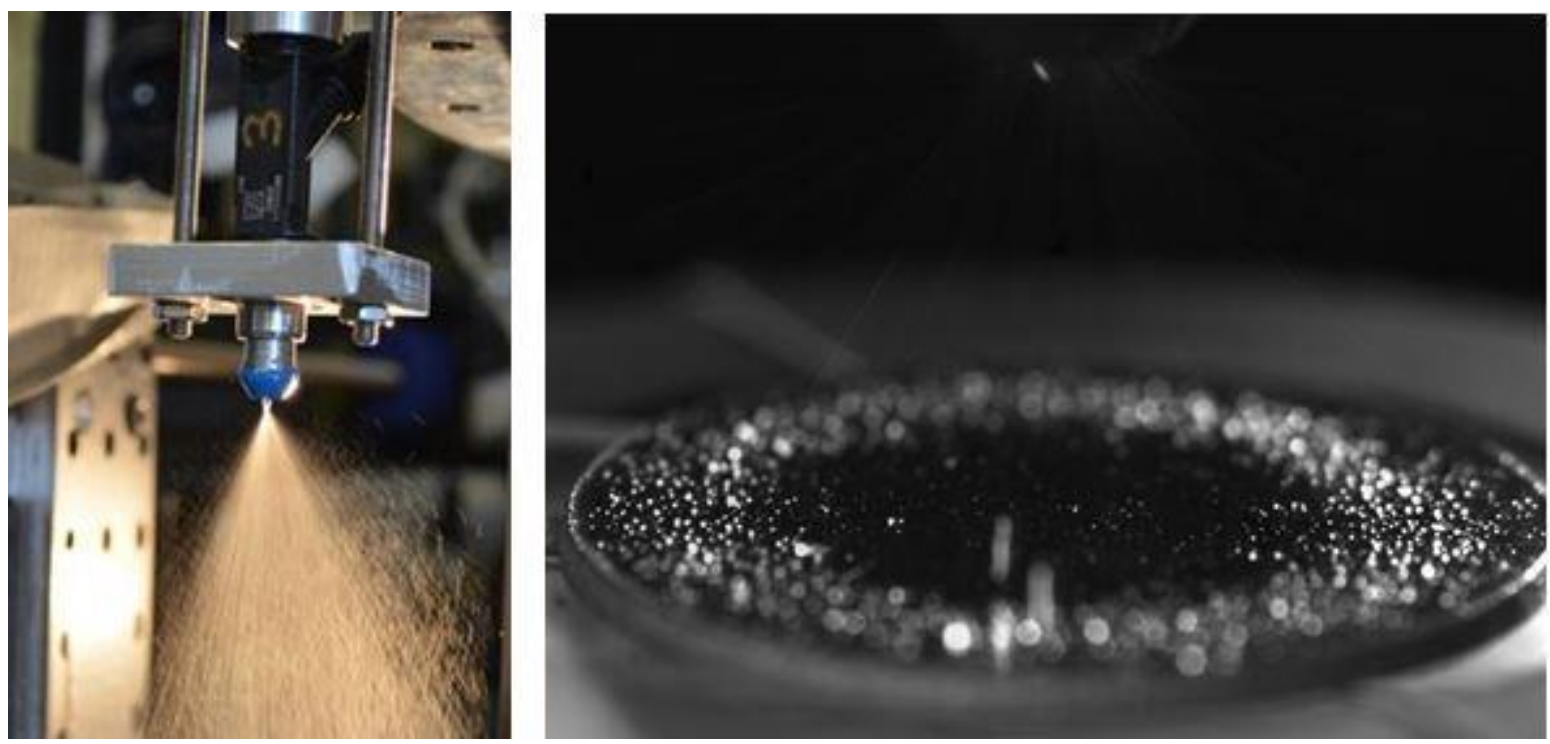

Figure 7 Comparison of In-Air Pattern to In-Vacuum Pattern 
3.3 Water Utilization Efficiency. The objective of the pulse spray is to impinge onto a surface for cooling. For a given pulse, some of the droplets in the spray will bounce off the disc rather than stick. In addition, some of the droplets have a curved trajectory and miss the disc. Figure 8 is a high speed video frame with $1 \mathrm{~ms}$ exposure to show the pathlines of the droplets. A larger curvature can be seen for the portion of the pathline near the disc. The curved trajectory is caused by the evaporating vapor leaving the disc as well as the evaporating vapor leaving the droplets that are in-flight from the nozzle to the disc. Most of the evaporated vapor flow is likely coming from the water mass that is sublimating at the disc. A much lesser amount is due to the evaporated flow from the flying droplets. Thus, the angle of curvature for the droplet path is more severe near the disc. Overall, only a small portion of the droplets miss the disc.

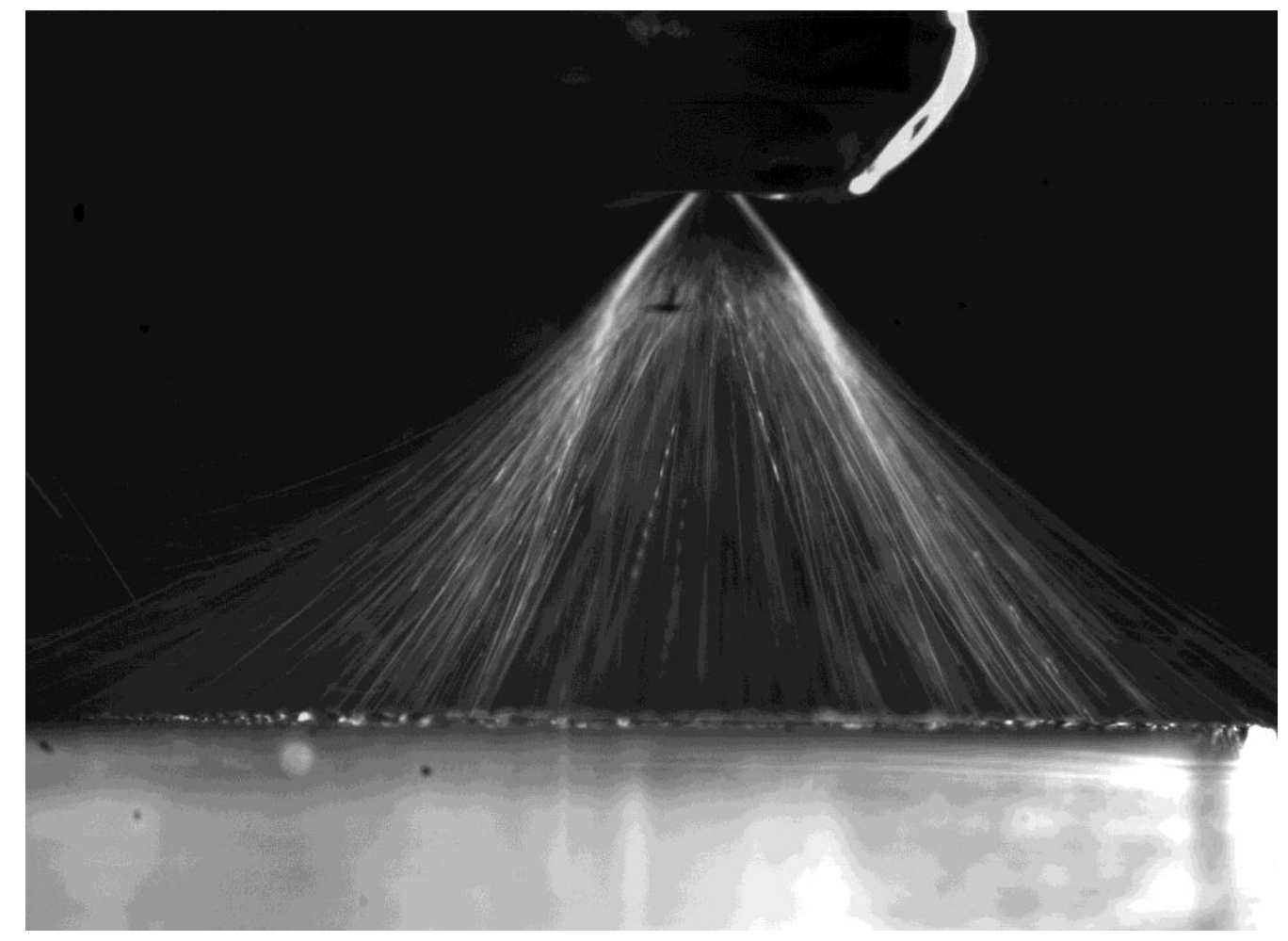

Figure 8 Observation of Droplet Pathlines with Curvature

These effects produce a cooling ability of the spray pulse that is somewhat less than would be assumed if the entire water mass from the pulse were available for phase change and disc cooling. This ratio of actual to ideal cooling ability of a spray is referred to as utilization efficiency, $\boldsymbol{\eta}$.

The evaluation of the amount of water deposited on the disc is made by the heat transfer consideration. In this research, the initial disc temperature is known. Also, the final disc temperature after all the water has evaporated is also measured. The total vaporization of deposited water takes about one second. Since there is no other major source of cooling in such a short time, then this entire temperature change is mainly due to the amount of water that had sublimated on the disc. Thus, the actual amount of water that landed on the disc can be deduced.

The amount of water that has left the nozzle is also known from measurements in air for the nozzle at the same nozzle pressure difference as in the vacuum. Tests in air were made with the pressure gauge reading of $25 \mathrm{psig}$, while tests in vacuum were made with a gauge pressure of $10 \mathrm{psig}$. This assured that the pressure differential across the nozzle was the same in both cases. The assumption that the nozzle driving pressure results in the same flowrate in air as in vacuum is valid, since the flow resistance across the solenoid, nozzle and swirl chamber is much larger than any flow resistance due to sheer against the air at the nozzle exit. The 
real vacuum pressure was 0.04 Torr ( 8 E -04 psia), which can be assumed essentially zero for flowrate/nozzle pressure measurements. For the spray mass during one pulse, a simple manual measurement method determined the amount of spray mass per second as a function of nozzle pressure. Then, knowing the opening and closing times of the nozzle in vacuum via the high speed video, the amount of mass during a pulse is known.

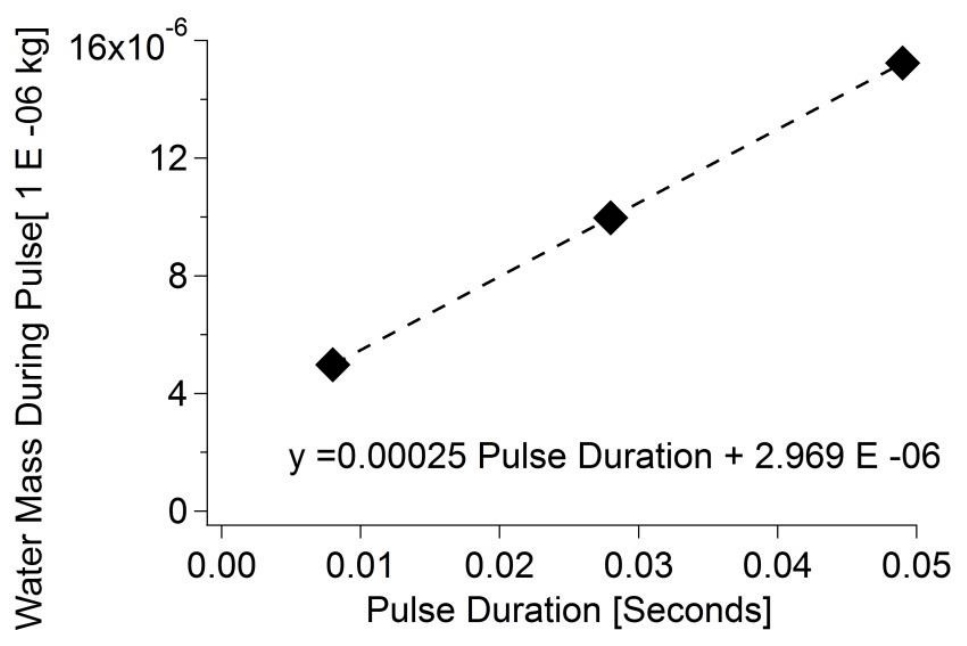

Figure 9 Measured Water Mass as a Function of Pulse Duration

Figure 9 shows the deduced water ejection in a pulse at different pulse durations. This linear fit indicates the consistency of the results of different pulse durations, but the extrapolation to zero pulse duration suggests that some of the mass, $3.0 \mathrm{E}-06 \mathrm{~kg}$, is remaining in the nozzle for each pulse. This volume of this liquid is about $3 \mathrm{~mm}^{3}$ which corresponds to the approximate volume of the swirl chamber and the space anterior to the nozzle "button" filter.

The amount of water deposited on the disc can be evaluated from the energy balance based upon the disc temperature changes through a pulse, evaluated according the heat required to cool and vaporize the deposited water as shown in Equation 1. Since the amount of mass during a spray pulse is directly measured and therefore known, and the amount of mass that actually reached the disc can be deduced from the total disc temperature change, the water utilization efficiency can be calculated.

$$
\eta=\frac{\text { Spray Mass Deduced from Disc Temperature Change }}{\text { Spray Mass Measured During a Pulse }}
$$

In Figure 10, a curve fit of the data (Equation 2) shows a power relationship between water utilization efficiency and pulse duration. For the three pulse durations presented, $(0.008,0.028,0.049)$ seconds, the water utilization efficiency is $(0.48,0.62,0.73)$. 


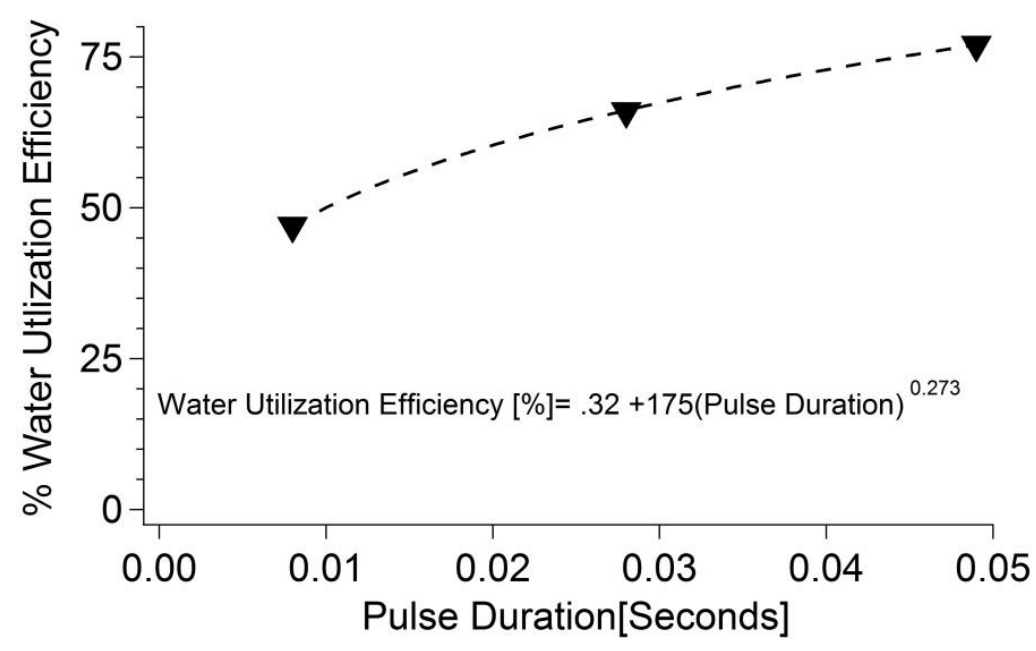

Figure 10 Water Utilization Efficiency as a Function of Pulse Duration

$$
\eta=0.32+175\left(t_{\text {pulse }}\right)^{0.273}
$$

The water contact angle on the gold plated copper disc has been measured as $47^{\circ}$. When water drops imping on this surface, there is a chance of bouncing. The reason for the increase in efficiency with pulse duration is that the longer pulse durations the surface have more wetted area. The wetted area intercepts incoming droplets, causing a sticking effect, and therefore allows for less bounce. More dry patches of disc exist in the shorter pulse cases. A dry surface is more susceptible to causing incoming droplets to bounce.

\section{CONCLUSIONS}

The experimental results show that a spray in air appears to be different from that in vacuum in two ways.

First, the startup in air produces an initial mass of liquid that is not yet atomized, and good atomization is delayed, whereas the startup in vacuum appears to show no initial mass, and immediate atomization. This could possibly be explained by the fact that with the ambient pressure startup, a small of amount of mass is resident in the nozzle and is slowly ejected at the start of the spray, as the inertia of this mass provides a flow resistance to the stream of liquid. In contrast, for a spray in vacuum, that small amount of mass would have already evaporated or sublimated before the initial spray startup. If this is true, then the swirl chamber is under vacuum conditions and void of any liquid. In the vacuum case, the liquid emerging from the pintle valve would then encounter an empty swirl chamber and no similar flow resistance. Then, the liquid inside the swirl chamber would easily develop the necessary velocity for good atomization before exiting the nozzle orifice. As a result, in the vacuum applications, sprays with short pulses are very feasible.

Second, vacuum operation can lower the water utilization efficiency, because the trajectory of the droplets leaving the nozzle is not a straight line, but bows outward away from the disc. It appears that the evaporating vapor leaving the surface entrains the droplets, which may cause the observed curved trajectory. In addition, if the contact angle of water on the solid surface is not extremely small, the drop bouncing could occur. For a short pulse, due to the larger dry area, the bouncing and loss of water deposition could be more significant than those of long pulses, where the drops are intercepted by the wetted area on the surface. 


\section{REFERENCES}

[1] O'conner, E., Zampiceni, J., Cerna, N., and Fuller, M., 1997, "Orbiter Flash Evaporator: Flight Experience and Improvements," SAE paper number 972262.

[2] Lethbridge, C., 1988, Sts-26 Fact Sheet, July 19, 2012, http://spaceline.org/shuttlechron/shuttlests26.html

[3] Hoffman, D. M., Singh, B., and Thomas, J. H., 1998, Handbook of Vacuum Science and Technology, Academic Press.

[4] Panao, M. R. O., 2009, "Intermittent Spray Cooling: A New Technology for Controlling Surface Temperature," International Journal of Heat and Fluid Flow, 30, pp. 117-130. 